\title{
Adolescents' conceptualisations of depression (a helpful label, chronic medical problem, or personality characteristic) affected their attitudes and decisions about treatment
}

Wisdom JP, Green CA. "Being in a funk": teens' efforts to understand their depressive experiences. Qual Health Res 2004; 14:122738.

\section{$Q$ How do adolescents with depression experience and understand the disease? How do they interpret the medical diagnosis?}

\section{DESIGN}

Modified grounded theory.

\section{SETTING}

Oregon and Washington state, USA.

\section{PARTICIPANTS}

A purposive sample of 15 adolescents 14-19 years of age (mean age $16 \mathrm{y}, 53 \%$ girls) who were diagnosed with depression was recruited from a non-profit, group model, health maintenance organisation. Most were no longer receiving treatment.

\section{METHODS}

13 adolescents participated in individual 90 minute interviews; 2 were interviewed by telephone. Questions focused on reasons for depression and the process of examining their feelings and determining whether they needed professional help. Interviews were tape recorded, and field notes documented additional information such as non-verbal communication. Tapes and field notes were transcribed verbatim, and data were analysed using constant comparison.

\section{MAIN FINDINGS}

A theoretical, chronological scheme was developed based on participants' descriptions of their experiences. (1) Growth of distress. Many teens wished to return to the time before they became depressed. They described the slow growth of distress over months or years, from imperceptible to incapacitating. Often, a specific event (eg, parental divorce or death of a relative or friend) triggered the growth of distress and exacerbated the normal difficulties of adolescence. Despite attempts to normalise their symptoms, teens were frustrated by so called "supportive" messages that denied their depression and expectations that they should be having the "time of their lives." (2) Being in a funk. Although specific symptoms and their severity varied, most participants described being in a funk in terms of low energy, a desire to be alone, and a pessimistic outlook. Other aspects included mistrusting their own perceptions. (3) Considering whether they are depressed. Attempts to normalise their experiences by comparing them with those of other teens became ineffective when symptoms (eg, suicidal thoughts) could no longer be characterised as "normal" or when duration of funks became too long. Participants eventually considered depression as a possible explanation for their experiences and talked with friends or family members who had been depressed to determine if they were actually depressed. (4) Receiving the diagnosis. Participants reported 3 types of reactions to receiving a diagnosis of depression. Labelers $(\mathrm{n}=6)$ perceived diagnosis as confirmation of their distress and felt relief. Labelers saw depression as a temporary situation and were interested in seeking out and trying self help interventions to reduce symptoms. For medicalisers $(\mathrm{n}=6)$, diagnosis confirmed they had a mental illness and required medical treatment. They felt ownership of the diagnosis and were distressed by it. They relied on healthcare providers for information and treatment options. Depression was seen as interfering with their lives but not something that required aggressive treatment. For identity infusers $(\mathrm{n}=3)$, a diagnosis of depression For correspondence: Dr J P Wisdom, Oregon Health and Science University, and Oregon Health Policy Institute, Portland, OR, USA. wisdomj@ohsu.edu Source of funding: Agency for Health Care Policy and Research. confirmed a part of their identity: being depressed was a personality characteristic, and thus they were more pessimistic about the likelihood of recovery. They did not try different symptom relief techniques and viewed interventions with suspicion and hopelessness. (5) Making sense of depression. Labellers accepted the diagnosis as a useful way of describing their feelings, a temporary life challenge, but not part of their identity. They tended to be treatment compliant but only for short periods. Medicalisers saw depression as something that belonged to them. They felt that healthcare providers were responsible for "fixing" the problem, and they were often non-compliant with treatment. Identity infusers agreed with the diagnosis and immediately integrated it into their self image. Some labellers and identity infusers considered depression as a learning experience, viewing symptoms as normal reactions to external stressors or indications of a need to learn something.

\section{CONCLUSIONS}

Adolescents' subjective experiences and conceptualisations of depression (ie, a helpful label, chronic medical problem, or personality characteristic) affected their attitudes and decisions about seeking medical treatment.

Commentary

A Ithough depression is the most common diagnosis of teenagers seeking mental health care, the number in treatment is well below the prevalence of the disorder in this age group. ${ }^{1}$ The study by Wisdom and Green provides a useful perspective on teenagers' understanding and response to a diagnosis of depression. The authors describe 3 specific clinical implications based on the findings of their study. Firstly, because study participants sought input from parents and peers about their feelings, it is critical that healthcare providers (ie, school nurses, paediatricians, and nurse practitioners) communicate accurate information about the disorder to parents and teens. This can be achieved through community education programmes, educational print and web based materials, and informal interactions during healthcare visits. Secondly, healthcare providers who have long term or consistent contacts with teens need to diligently assess changes in behaviour that might indicate the beginning of distress, which could lead to clinical depression. Thirdly, because teens' understanding and response to depression affect their decisions and attitudes about seeking treatment, the theoretical schemes proposed might help shape the individualised treatment approaches of healthcare providers. However, the authors caution that using categories to label responses can have negative effects. For example, teens labelled as "identity infusers" might conclude that the development of all the associated attributes in this category is somehow inevitable. These teens may then become pessimistic about their recovery.

The primary purpose of the study by Wisdom and Green was to initiate discussion about teens' experiences with depression and design a theoretical framework for further research on the topic. The 3 categories provide such a framework. Although the category scheme does have the potential to improve the prevention, identification, and treatment of depression in teenagers, more research is needed to assess its validity in a larger sample of the teen population.

Lynda Slimmer, RN, PhD University of Illinois at Chicago Chicago, Illinois, USA

1 Hirschfeld RM, Keller MB, Panico S, et al. The National Depressive and Manic-Depressive Association consensus statement on the undertreatment of Manic-Depressive Association consensus
depression. JAMA 1997;277;333-40. 